\title{
No Significance of the Free Cortisol Index Compared to Total Cortisol in Critically Ill Patients
}

\author{
Kyung Won Kim ${ }^{1,2}$, Sang Wan Kim ${ }^{1,3}$, Hee Joung Kim ${ }^{1}$, Chan Soo Shin ${ }^{1}$, Sung Jae Park ${ }^{4}$, Gil Joon Suh ${ }^{5}$, Seong Yeon Kim ${ }^{1}$ \\ Department of Internal Medicine ${ }^{1}$, Seoul National University College of Medicine, Seoul; Healthcare System Gangnam Center ${ }^{2}$, Seoul National \\ University Hospital, Seoul; Department of Internal Medicine ${ }^{3}$, Seoul National University Boramae Hospital, Seoul; Seoul National University \\ Hospital Clinical Research Institute ${ }^{4}$, Seoul; Department of Emergency Medicine 5 , Seoul National University College of Medicine, Seoul, Korea
}

Background: Some patients exhibit an inadequate response of cortisol to stressful conditions; this state is known as critical illnessrelated corticosteroid insufficiency. These patients have low serum binding protein concentrations, thereby suggesting that total serum cortisol may not be reflective of circulating cortisol activity. As the free cortisol index (FCI = total cortisol/corticosteroid-binding globulin) has been correlated with serum free cortisol, we measured FCI in Korean patients for the first time. In this study, we attempted to determine whether FCI was superior to total cortisol in predicting 30-day mortality.

Methods: We recruited 65 critically ill patients with relatively high Acute Physiology, Age and Chronic Health Evaluation (APACHE III) scores. Fourteen patients with pituitary disease but normal pituitary function were recruited from outpatient clinics. Total cortisol and corticosteroid-binding globulin were measured in patients and controls during the short Synacthen test.

Results: The basal cortisol level and basal FCI level were higher in patients $(\mathrm{n}=65)$ than in healthy controls $(P<0.001$, respectively). We found that total cortisol was strongly correlated with FCI $(P<0.001)$ in critically ill patients; however, neither total cortisol nor FCI were associated with 30-day mortality among patients. Only severe clinical criteria (such as APACHE-III scores and low albumin) were associated with 30-day mortality.

Conclusion: Our results do not suggest that FCI is more accurate than total cortisol in predicting clinical outcomes in critically ill patients. (Endocrinol Metab 26:120-125, 2011)

Key Words: Critical illness, Adrenal insufficiency, APACHE, Mortality, Free cortisol index (FCI)

\section{INTRODUCTION}

Cortisol must be adequately secreted under stressful conditions because it is one of the essential hormones for maintaining and restoring homeostasis following stressful events [1]. Acute physiologic stress, e.g., septic shock, increases adrenocorticotropic hormone (ACTH) and cortisol secretion through activation of the hypothalamus-pituitary-adrenal (HPA) axis. However, some patients fail to mount an adequate cortisol response to overwhelming stress, and this inadequate response is referred to as "critical illness-related corticosteroid insufficiency (CIRCI)".

Although it is generally agreed that adrenal failure may be com-

Received: 25 July 2010, Accepted: 13 December 2010

Corresponding author: Seong Yeon Kim

Department of Internal Medicine, Seoul National University College of Medicine, 101 Daehak-ro, Jongno-gu, Seoul 110-744, Korea

Tel: +82-2-2072-3216, Fax: +82-2-762-9662, E-mail: seongyk@plaza.snu.ac.kr

* This study was supported by a grant (04-2005-031-0) from the Seoul National University Hospital Research Fund. mon in subgroups of critically ill patients and should be treated immediately [2], the diagnosis and management of this disorder remains controversial. This controversy is based on several facts. First, there is no gold-standard tool for diagnosis of CIRCI. Ideally, insulin tolerance test is used for assessing the entire axis, but this test is inappropriate in the critical care setting. Thus, investigations are limited to measurements of basal cortisol levels or the short Synacthen test (SST). Many criteria have been suggested for defining CIRCI, yet this issue is still being debated $[1,3,4]$. However, the SST has been criticized that sepsis itself may evoke maximum cortisol secretion in individuals, thus SST may not be needed in maximally stimulated situation such as sepsis. Second, it assesses adrenal gland function directly, thereby potentially missing the diagnosis of adrenal failure secondary to hypothalamic or pituitary failure. Third, the total cortisol level, which is used in most criteria, does not provide an adequate assessment of adrenal cortical function. Over $90 \%$ of circulating cortisol is bound to corticosteroid-binding globulin (CBG) with less than 10\% in the free, biologically active 
form [2]. Considering CBG levels decrease during critical illness, measurement of total cortisol concentrations can be underestimated. Free cortisol concentrations provide a more reliable indicator, but they are relatively difficult to measure in the clinical setting, and the normal range of free cortisol in critically ill patients is currently unclear. To overcome these limitations, the free cortisol index (FCI, serum cortisol divided by serum CBG concentration) was suggested to reflect the free cortisol. Forth, the benefit of glucocorticoids in sepsis is still unclear $[1,5]$. If there were a more confirmative diagnosis, the benefit would be greater.

Thus, it is important to define CIRCI to provide adequate treatment. As FCI has been shown to correlate with serum free cortisol $[6,7]$ and can be measured more easily, we measured FCI in Korean patients for first time in this study. In this study, we investigated whether FCI was correlated with total cortisol, and was superior to total cortisol or not in predicting the clinical outcome. Although, sepsis itself may evoke maximum cortisol secretion in individuals, many studies used the SST to evaluate relative adrenal insufficiency (RAI). Thus, we tested the SST in critically ill patients to compare our results with others. In addition, we evaluated which guidelines were better for diagnosing RAI. Although this study was not aimed to evaluate the efficacy of glucocorticoid supplementation, we evaluated the prognostic value of certain criteria on RAI diagnosis.

\section{MATERIALS AND METHODS}

This study was conducted between November 2005 and December 2006 in the Emergency Intensive Care Unit of the Seoul National University Hospital. The study protocol was approved by the Seoul National University Hospital Ethics Committee, and written informed consent was obtained from patients or their legal guardians.

\section{Patients}

We recruited 65 critically ill patients with higher Acute Physiology, Age and Chronic Health Evaluation (APACHE III) score ( $\geq 15$ ) [8]. The APACHE III score was assessed using the worst clinical parameters within 24 hour after admission to the intensive care unit. Patients were excluded if they had a history of hypothalamic-pituitary or adrenal disease, if they had taken glucocorticoids or estrogen in the preceding year or medications known to influence glucocorticoid secretion (e.g., ketoconazole) in the preceding six months, or if they were pregnant or breast-feeding. Patients with cirrhosis, irreversible underlying disease such as advanced malignancy or AIDS, pulmonary embolism, and acute myocardial infarction were also excluded. The patients were divided into three groups according to the following states: sepsis, septic shock, or other disease. Definitions of septic shock and sepsis followed the American College of Chest Physicians/Society of Critical Care Medicine Consensus Conference Committee criteria [9]. Septic shock is defined as the presence of systemic inflammatory response syndrome (SIRS), documented infection or positive blood culture and organ dysfunction in addition to hypotension despite adequate fluid resuscitation. Sepsis is defined as SIRS that has proven or suspected microbial etiology. SIRS is defined by the presence of two or more of the following criteria: fever (temperature $>38^{\circ} \mathrm{C}$ ) or hypothermia (temperature $<36^{\circ} \mathrm{C}$ ), tachycardia ( $>90$ beats $/ \mathrm{min}$ ), tachypnea ( $>20$ breaths/min), leucocytosis or leucopenia (white blood cell count $>12,000$ or $<4000$ cells $/ \mu \mathrm{L}$ ), or immature neutrophils (bands $>10 \%$ ). The criteria for organ dysfunction or a hypoperfusion abnormality included the following: altered mental status, hypoxemia $\left(\mathrm{PaO}_{2}<75 \mathrm{mmHg}\right)$, oliguria (urine output $<30$ $\mathrm{mL} / \mathrm{h}$ ), or elevated plasma lactate level (> $2 \mathrm{mmol} / \mathrm{L})$. The definition of hypotension was a systolic blood pressure $<90 \mathrm{mmHg}$ or a reduction in systolic blood pressure of $40 \mathrm{mmHg}$ or more from baseline. The patients with other diseases were those who did not meet the criteria of sepsis or septic shock, needed intensive care, and had a high APACHE III score. The category of other diseases consists of pneumonia, bleeding, pulmonary edema, trauma, rapidly progressive glomerulonephritis, rhabdomyolysis, electrolyte imbalances.

\section{Control}

Fourteen patients with pituitary disease but a normal pituitary function were recruited from outpatient clinics. They had had pituitary diseases before, but they were cured after optimal treatment. The controls had received no medication and had no evidence of any other endocrine or cancer, renal or liver dysfunction or infection. Insulin tolerance tests were performed after an overnight fast on pituitary controls using regular insulin (0.1-0.2 U/kg i.v.). They showed normal GH and cortisol responses after the stimulation test (stimulated GH $>5 \mu \mathrm{g} / \mathrm{L}$, stimulated cortisol $>18 \mu \mathrm{g} / \mathrm{dL}$ ).

\section{Clinical evaluation}

Information relating to: 1) age and gender, 2) infection site(s) and 
organisms, 3) severity of illness at baseline using the APACHE III scoring system, and 4) disease outcome including mortality were collected.

\section{Laboratory measurements}

An SST was performed while patients still met the inclusion criteria. Most patients (89\%) performed the SST within 48 hour after admission to the intensive care unit. Blood samples were collected immediately before and 30 and 60 minutes after Synacthen $(250 \mu \mathrm{g}$ ACTH 1-24, Synacthen, CIBA, West Sussex, United Kingdom) was administered. Measurements of serum total cortisol were performed with the use of a standard radioimmunoassay (CIS BIO international CORT-CT2 kit, France). The intra- and inter-assay coefficients of variation were 4.3 and $4.2 \%$, respectively. CBG was analyzed by a commercial radioimmunoassay (CBG-RIA kit: Biosource Europe S.A., Nivelles, Belgium). This assay has a stated imprecision of generally less than $7 \%$ for between and within assay variation and a detection limit of $120 \mathrm{nmol} / \mathrm{L}(0.25 \pm 0.02 \mu \mathrm{g} / \mathrm{mL})$. FCI was calculated from the following equation: Total cortisol (nmol/L)/ CBG (mg/L).

Samples from individual subjects were analyzed together. Samples were tested in duplicate.

\section{Statistical analysis}

Spearman rank correlation was used to assess whether there was a relationship between basal cortisol and basal FCI in the pituitary control group. In the patient group, Pearson correlation was used. The plasma cortisol increment was defined as the difference be- tween the basal cortisol and the highest concentration after the SST. Descriptive statistics are reported as the median score (interquartile range). Frequency comparisons were made with the chisquared test or Fisher's exact test. Two-group comparisons of normally distributed data were performed by Student's $t$-test. For data not normally distributed, the Mann-Whitney $U$ test was used only if two groups were compared, and Kruskal-Wallis one-way analysis of variance was used if more than two groups were being compared. Statistical significance was set at $P<0.05$. Statistical analyses were performed using the SPSS for Windows release 17 (SPSS, Chicago, IL, USA).

\section{RESULTS}

\section{Control and patient groups}

The median age of the controls was 48.5 (37-53) years, and they were younger than the patient group $(P<0.001)$ (Table 1$)$. The basal cortisol level was $8.6(6.3-10.2) \mu \mathrm{g} / \mathrm{dL}$, which was lower than that of the patient group $(P<0.001)$. In the control group, basal cortisol and basal FCI were well correlated with each other (Spearman rank correlation coefficient $=0.73, P=0.003$ ). In the patient group, basal cortisol and basal FCI were also well correlated (Pearson correlation coefficient $=0.71, P<0.001$ ). The level of CBG was lower in the patient group $(P=0.005)$, accordingly basal FCI was higher in patients group $(P<0.001)$. Patients with septic shock had higher APACHE III scores $(P=0.013)$ and lower plasma albumin levels $(P=0.056)$ than patients with sepsis. Patients with septic shock had higher APACHE scores $(P=0.007)$, lower plasma albu-

Table 1. Comparison of basal characteristics amongst the patient and control groups

\begin{tabular}{|c|c|c|c|c|c|}
\hline & C & & Patient & $=65)$ & \\
\hline & & Total $(n=65)$ & Septic shock $(n=29)$ & Sepsis $(n=19)$ & Other $(n=17)$ \\
\hline Age (years) ${ }^{*}$ & $48.5(37-53)$ & $68(5.85-76.0)$ & $68(61.5-76.5)$ & $65(48-75)$ & $68(61.5-76)$ \\
\hline Albumin (g/dL) & $4(3.8-4.23)$ & $2.7(2.3-3.2)$ & $2.4(2.2-2.9)$ & $3.0(2.3-3.3)$ & $3.1(2.5-3.5)$ \\
\hline APACHE-III ${ }^{\dagger}$ & Not available & $58.0(46.5-72.5)$ & $63.0(55.0-92.0)$ & $52.0(31.0-67.0)$ & $54.0(19.5-66.0)$ \\
\hline Basal cortisol $^{*}(\mu \mathrm{g} / \mathrm{dL})$ & $8.6(6.3-10.2)$ & $15.4(11.8-24.6)$ & 20.1 (12.4-29.8) & $14.4(11.5-23.4)$ & $14.2(9.2-18.1)$ \\
\hline Basal CBG* $(\mu \mathrm{g} / \mathrm{mL})$ & $41.3(33.8-46.9)$ & $32.5(21.3-38.8)$ & $35.0(27.5-40.0)$ & $35(25.0-42.5)$ & $22.5(17.5-32.5)$ \\
\hline Basal FCl ${ }^{*}(\mathrm{nmol} / \mathrm{mg})$ & $5.9(4.2-8.8)$ & $16.9(10.4-23.0)$ & $17.1(11.7-26.2)$ & $12.6(8.7-25.8)$ & $19.6(9.5-22.6)$ \\
\hline Peak cortisol ( $\mu \mathrm{g} / \mathrm{dL})$ & $25.5(21.6-27.7)$ & $22.7(18.0-33.7)$ & $25.2(18.1-42.2)$ & $19(25.7-34.1)$ & $22.1(14.8-23.6)$ \\
\hline $\mathrm{FCl}$ of peak cortisol ${ }^{*}(\mathrm{nmol} / \mathrm{mg})$ & $19.5(17.3-24.0)$ & $20.9(14.8-38.3)$ & $23.6(14.8-41.8)$ & $18.9(13.4-41.9)$ & $24.6(16.9-34.8)$ \\
\hline 30-day mortality & 0 & $10(15.4 \%)$ & $8(27.6 \%)$ & $1(5.3 \%)$ & $1(5.9 \%)$ \\
\hline
\end{tabular}

The table shows means median score (interquartile range).

${ }^{*} P<0.05$ between control group and total patients. ${ }^{\dagger} P<0.05$ between septic shock patients and sepsis patients.

APACHE-III, Acute Physiology, Age and Chronic Health Evaluation-III score; CBG, corticosteroid-binding globulin; FCI, free cortisol index. 
min levels $(P=0.036)$, higher basal cortisol levels $(P=0.044)$, and lower basal and 30 min CBG levels $(P=0.024$ and $P=0.034)$ than patients with other diseases. There was a linear correlation between total cortisol (or basal FCI) and higher APACHE III scores ( $P$ $=0.006$ and 0.075 , respectively), not by clinical grouping (cortisol in septic shock vs. sepsis vs. other disease, $P=0.117$ ).

Table 2 shows the characteristics of survivors and non-survivors. APACHE III scores and albumin levels were significantly different between these groups. In the non-survivor group, the percentage of septic shock patients was higher than in the survivor group ( $P=$ 0.05). Neither cortisol nor FCI were predictable for mortality. The frequencies of critical illness-related corticosteroid insufficiency (CIRCI) and association with mortality

Table 3 shows 30-day in-hospital mortality according to currently suggested criteria. The mortality rate was $15.4 \%$ (10/65). Using the

Table 2. Short Synacthen test results according to 30-day hospital mortality

\begin{tabular}{lccc}
\hline & $\begin{array}{c}\text { Survivors } \\
(\mathrm{n}=55)\end{array}$ & $\begin{array}{c}\text { Non-survivors } \\
(\mathrm{n}=10)\end{array}$ & $P$ \\
\hline Age (years) & $68(57-75)$ & $71(66-81.3)$ & 0.075 \\
APACHE-III & $56(42-67)$ & $79(67.5-100.5)$ & 0.001 \\
Septic shock patients & $12(38.2 \%)$ & $8(80 \%)$ & 0.05 \\
Albumin $(\mathrm{g} / \mathrm{dL})$ & $2.7(2.4-3.3)$ & $2.3(2.1-2.9)$ & 0.024 \\
Basal Cortisol $(\mu \mathrm{g} / \mathrm{dL})$ & $15.4(11.8-23.4)$ & $18.2(8.9-28.8)$ & 0.86 \\
Cortisol at T30 $(\mu \mathrm{g} / \mathrm{dL})$ & $21.5(15.6-30.3)$ & $20(14.4-33.1)$ & 0.87 \\
Cortisol at T60 $(\mu \mathrm{g} / \mathrm{dL})$ & $24(18.3-33.2)$ & $20.2(13.0-33.1)$ & 0.51 \\
Peak cortisol $(\mu \mathrm{g} / \mathrm{dL})$ & $24.3(18.3-34.1)$ & $20.8(14.5-34.1)$ & 0.59 \\
$\Delta$ max cortisol $(\mu \mathrm{g} / \mathrm{dL})$ & $7.6(3.3-11.7)$ & $2.3(0.5-8.8)$ & 0.16 \\
Basal FCl $(\mathrm{nmol} / \mathrm{mg})$ & $16.9(11.0-21.9)$ & $18.6(8.7-51.9)$ & 0.46 \\
FCl at T30 $(\mathrm{nmol} / \mathrm{mg})$ & $21.0(13.6-34.5)$ & $18.6(13.4-35.9)$ & 0.83 \\
FCl at T60 $(\mathrm{nmol} / \mathrm{mg})$ & $22.5(15.6-41.9)$ & $20.9(12.8-46.7)$ & 0.86 \\
Peak FCl $(\mathrm{nmol} / \mathrm{mg})$ & $23.6(15.6-38.9)$ & $20.1(9.4-42.4)$ & 0.59 \\
$\Delta \mathrm{FCl}(\mathrm{nmol} / \mathrm{mg})$ & $7.6(2.7-13.4)$ & $-0.9(-4.3-13.4)$ & 0.18 \\
\hline
\end{tabular}

The table shows means median score (interquartile range).

T60, 60 min after the short Synacten test; T60, 60 min after the short; Synacthen test; $\Delta$ max, peak cortisol minus baseline cortisol. currently most-validated criterion [4,10], the frequency of RAI ( $\Delta$ cortisol <9) was 58.5\% (38/65). The cut-off level 18 was used because a normal response was defined by a peak plasma cortisol of greater than $18 \mu \mathrm{g} / \mathrm{dL}$. The rates of RAI were 22-69\%, although there was no difference in mortality between the groups. Although septic shock patients were more likely to die, certain specific RAI criteria failed to reliably predict 30-day mortality. To analyze the mortality difference according to CIRCI in similar clinical condition, we divided patients into 2 groups stratified by APACHE III score (less severe APACHE group [score $\leq 58, \mathrm{n}=33$ ] and more severe [APACHE3 score $>58, \mathrm{n}=32$ ] group). There was no mortality difference between CIRCI and non-CIRCI in similar clinical condition. The mortality of CIRCI was not affected by sepsis, septic shock and other disease, either (data not shown).

\section{DISCUSSION}

We planned the present study to determine whether FCI is well correlated with total cortisol and provides more information for identification of patients who would benefit from corticosteroid treatment during critical illness. We found that basal total cortisol correlated well with basal FCI $(P<0.001)$ in critically ill patients, which means the patients with low total cortisol were more likely to have low FCI. Clinical severity resulted in higher APACHE III scores, lower albumin concentration, and higher basal cortisol; however, only two of the former factors were associated with 30day mortality. Accordingly, FCI did not provide any additional information for predicting patient 30-day mortality. The prevalence of RAI was 22-69\% according to currently suggested criteria $[3,4,10,11]$; none of these criteria was associated with 30-day mortality and we could not suggest which guideline was best for predicting in 30-day mortality in the present study.

Activation of the HPA axis is one of several important responses

Table 3. 30-day mortality difference among the patient groups according to various critical illness-related corticosteroid insufficiency (CIRCI) criteria

\begin{tabular}{|c|c|c|c|c|c|}
\hline & $\begin{array}{l}\text { Septic shock } \\
\quad(n=29)\end{array}$ & $\begin{array}{l}\text { Sepsis } \\
(n=19)\end{array}$ & $\begin{array}{l}\text { Other } \\
(n=17)\end{array}$ & $\begin{array}{c}\text { Total } \\
(n=65)\end{array}$ & $P$ \\
\hline Basal cortisol <18 $\mu \mathrm{g} / \mathrm{dL}(\mathrm{n}=38)[3]$ & $4 / 13(31 \%)$ & $0 / 12(0 \%)$ & $1 / 13(8 \%)$ & $5 / 38(13 \%)$ & 0.058 \\
\hline Any cortisol $<18 \mu \mathrm{g} / \mathrm{dL}(\mathrm{n}=15)$ & $2 / 7(29 \%)$ & $0 / 3(0 \%)$ & $1 / 5(20 \%)$ & $3 / 15(20 \%)$ & 0.585 \\
\hline$\Delta$ Cortisol $<9 \mu \mathrm{g} / \mathrm{dL}(\mathrm{n}=38)[3]$ & $6 / 18(33 \%)$ & $1 / 10(10 \%)$ & $1 / 10(10 \%)$ & $8 / 38(21 \%)$ & 0.212 \\
\hline Basal cortisol $<10 \mu \mathrm{g} / \mathrm{dL}$ or $\Delta$ Cortisol $<9 \mu \mathrm{g} / \mathrm{dL}(\mathrm{n}=43)$ [4] & $7 / 19(37 \%)$ & $1 / 11(9 \%)$ & $1 / 13(8 \%)$ & $9 / 43(21 \%)$ & 0.074 \\
\hline Basal cortisol $<15 \mu \mathrm{g} / \mathrm{dL}$ or $\Delta$ Cortisol $<9 \mu \mathrm{g} / \mathrm{dL}(\mathrm{n}=45)[4,10]$ & $6 / 18(33 \%)$ & $1 / 12(8 \%)$ & $1 / 15(7 \%)$ & $8 / 45(18 \%)$ & 0.083 \\
\hline $30 \mathrm{~min}$ free cortisol index $(<12 \mathrm{nmol} / \mathrm{mg})(\mathrm{n}=14)[11]$ & $2 / 5(40 \%)$ & $0 / 6(0 \%)$ & $0 / 3(0 \%)$ & $2 / 14(14 \%)$ & 0.122 \\
\hline
\end{tabular}

Comparison among patients with septic shock, sepsis, and other diseases was calculated using chi-squared tests. 
to stressful events and critical illnesses. As adrenal response is frequently disturbed in critically ill patients, glucocorticoid replacement may be beneficial to some patients. Although the benefit was confined to the group of SST nonresponders ( $\Delta$ cortisol $<100-250$ $\mathrm{nmol} / \mathrm{L})$, moderate-dose hydrocortisone (200-300 mg/d) improved the 28-day mortality in patients with septic shock [12,13]. However, retrospective Corticus cohort study and a meta-analysis of six randomized, controlled trials demonstrated greater shock reversal with hydrocortisone but no benefit in terms of mortality [4,5]. Thus, this results made clinicians reluctant to prescribe corticosteroids, though Corticus study was criticized for selection bias (patients least likely to benefit from corticosteroids were enrolled in the study) [14]. Potential benefits of glucocorticoid therapy in critically ill patients include anti-inflammatory effects, inhibition of cytokine production, decreased arachidonic acid synthesis, increased antiinflammatory cytokine expression, and effects on the cardiovascular system to promote both improved vascular tone and catecholamine responsiveness [1]. Adverse sequelae include hyperglycemia, insulin resistance, bone loss, muscle wasting, hypokalemia, and changes in mental status [1]. Considering the benefits of glucocorticoid, the use of moderate-dose hydrocortisone seems rational in patients with septic shock who have responded poorly to fluid resuscitation and vasopressor agents [4].

However, other studies reported that low total cortisol levels have been associated with favorable outcomes [5,15,16]. Although we could not find a significant difference in basal total cortisol and FCI concentrations between septic shock patients and sepsis patients, severe patients were more likely to have higher cortisol level. If more patients were enrolled in our study, we might have found a significant trend between the groups.

The rate of CIRCI in the present study was $58.5 \%$ (CIRCI was defined when $\Delta$ cortisol was less than $9 \mu \mathrm{g} / \mathrm{dL}$ ), which was higher than another Korean study [17]. However, we failed to show a mortality difference between CIRCI and non-CIRCI. It is still controversial as to whether cortisol could predict in-hospital mortality [18]. The mortality rate was similar in CIRCI groups defined by the various criteria in our study (Table 3). Although the same criteria were used for all groups, patients with shock were more likely to die than patients with sepsis or other diseases. As higher APACHE III scores reflect the seriousness of the clinical condition, the most important prognostic factor was clinical severity rather than CIRCI in the current study. However, the limitations of the present study are that a small sample size was used and patients were enrolled from a single institution; therefore, there was only a small number of inhospital deaths $(15.4 \%$, which is relatively lower than others, i.e., 31.5-61\%). This low death rate might be the reason why a significant correlation could not be detected between survival and the level of baseline serum cortisol or change in cortisol. Ethnic differences may account for this discrepancy as well.

Cortisol levels vary broadly with disease severity making it difficult to define appropriate responses. Indeed, only the free hormone is biologically active, whereas more than $90 \%$ of circulating cortisol is bound to proteins, predominantly CBG but also albumin [2]. Our control group was younger than patients' group and their clinical condition was much different to patients' group. As the cortisol secretion does not change during age [19], we speculate that the cortisol concentration of control group is similar to that of the aged. Also, it seems to be meaningful to assess the CBG concentration in normal population. Circulating CBG concentrations are approximately $700 \mathrm{nmol} / \mathrm{L}$ [2], and this is consistent with our results (our basal CBG concentration of control group was $41.3 \pm$ $10.5 \mu \mathrm{g} / \mathrm{mL}$, i.e., $794.2 \pm 20.2 \mathrm{nmol} / \mathrm{L}$ ). During acute illness, particularly sepsis, CBG levels fall by as much as $50 \%$, resulting in a significant increase in the percentage of free cortisol [20]. A recent study of critically ill patients suggested that low cortisol binding proteins, rather than low free cortisol levels, may underlie reports of reduced total cortisol in critical illness [21]. However, direct determination of free cortisol levels is labor-intensive and impractical for routine clinical use. CBG was decreased among patients with critical illness in our study, which is consistent with another study. Nevertheless, FCI was not superior to total cortisol in this study. FCI was linearly correlated with total cortisol in critically ill patients. Neither a change in cortisol nor a change in FCI provided additional information to predict mortality. As we did not try glucocorticoid supplementation in CIRCI patients, we cannot evaluate the efficacy of glucocorticoid therapy.

In the current study, calculating FCI, instead of total cortisol, did not help to distinguish between survivors and non-survivors. Therefore, calculation of free cortisol concentration, instead of total, most likely does not help to identify patients who could potentially benefit from glucocorticoid treatment.

\section{REFERENCES}

1. Venkataraman S, Munoz R, Candido C, Witchel SF: The hypothalamicpituitary-adrenal axis in critical illness. Rev Endocr Metab Disord 8:365- 
373, 2007

2. Stewart PM: Williams textbook of endocrinology. 11th ed. pp445-503, Philadelphia, Saunders, 2008

3. Arafah BM: Hypothalamic pituitary adrenal function during critical illness: limitations of current assessment methods. J Clin Endocrinol Metab 91:3725-3745, 2006

4. Marik PE, Pastores SM, Annane D, Meduri GU, Sprung CL, Arlt W, Keh D, Briegel J, Beishuizen A, Dimopoulou I, Tsagarakis S, Singer M, Chrousos GP, Zaloga G, Bokhari F, Vogeser M: Recommendations for the diagnosis and management of corticosteroid insufficiency in critically ill adult patients: consensus statements from an international task force by the American College of Critical Care Medicine. Crit Care Med 36:1937-1949, 2008

5. Lipiner-Friedman D, Sprung CL, Laterre PF, Weiss Y, Goodman SV, Vogeser M, Briegel J, Keh D, Singer M, Moreno R, Bellissant E, Annane D: Adrenal function in sepsis: the retrospective Corticus cohort study. Crit Care Med 35:1012-1018, 2007

6. le Roux CW, Chapman GA, Kong WM, Dhillo WS, Jones J, AlaghbandZadeh J: Free cortisol index is better than serum total cortisol in determining hypothalamic-pituitary-adrenal status in patients undergoing surgery. J Clin Endocrinol Metab 88:2045-2048, 2003

7. le Roux CW, Sivakumaran S, Alaghband-Zadeh J, Dhillo W, Kong WM, Wheeler MJ: Free cortisol index as a surrogate marker for serum free cortisol. Ann Clin Biochem 39:406-408, 2002

8. Knaus WA, Wagner DP, Draper EA, Zimmerman JE, Bergner M, Bastos PG, Sirio CA, Murphy DJ, Lotring T, Damiano A: The APACHE III prognostic system. Risk prediction of hospital mortality for critically ill hospitalized adults. Chest 100:1619-1636, 1991

9. American College of Chest Physicians/Society of Critical Care Medicine Consensus Conference: definitions for sepsis and organ failure and guidelines for the use of innovative therapies in sepsis. Crit Care Med 20:864874, 1992

10. Cooper MS, Stewart PM: Corticosteroid insufficiency in acutely ill patients. N Engl J Med 348:727-734, 2003
11. Dhillo WS, Kong WM, Le Roux CW, Alaghband-Zadeh J, Jones J, Carter G, Mendoza N, Meeran K, O'Shea D: Cortisol-binding globulin is important in the interpretation of dynamic tests of the hypothalamic-pituitaryadrenal axis. Eur J Endocrinol 146:231-235, 2002

12. Annane D, Sebille V, Charpentier C, Bollaert PE, Francois B, Korach JM, Capellier G, Cohen Y, Azoulay E, Troche G, Chaumet-Riffaud P, Bellissant $\mathrm{E}$ : Effect of treatment with low doses of hydrocortisone and fludrocortisone on mortality in patients with septic shock. JAMA 288:862-871, 2002

13. de Jong MF, Beishuizen A, Spijkstra JJ, Groeneveld AB: Relative adrenal insufficiency as a predictor of disease severity, mortality, and beneficial effects of corticosteroid treatment in septic shock. Crit Care Med 35:18961903, 2007

14. Marik PE, Pastores SM, Kavanagh BP: Corticosteroids for septic shock. N Engl J Med 358:2069-2070, 2008

15. Bendel S, Karlsson S, Pettila V, Loisa P, Varpula M, Ruokonen E: Free cortisol in sepsis and septic shock. Anesth Analg 106:1813-1819, 2008

16. Sam S, Corbridge TC, Mokhlesi B, Comellas AP, Molitch ME: Cortisol levels and mortality in severe sepsis. Clin Endocrinol (Oxf) 60:29-35, 2004

17. Kwon YS, Kang E, Suh GY, Koh WJ, Chung MP, Kim H, Kwon OJ, Chung JH: A prospective study on the incidence and predictive factors of relative adrenal insufficiency in Korean critically-ill patients. J Korean Med Sci 24:668-673, 2009

18. Gotoh S, Nishimura N, Takahashi O, Shiratsuka H, Horinouchi H, Ono H, Uchiyama N, Chohnabayashi N: Adrenal function in patients with community-acquired pneumonia. Eur Respir J 31:1268-1273, 2008

19. Lamberts SW: Williams textbook of endocrinology. 11th ed. pp1185-1199, Philadelphia, Saunders, 2008

20. Marik PE: Mechanisms and clinical consequences of critical illness associated adrenal insufficiency. Curr Opin Crit Care 13:363-369, 2007

21. Hamrahian AH, Oseni TS, Arafah BM: Measurements of serum free cortisol in critically ill patients. N Engl J Med 350:1629-1638, 2004 